\title{
An improved axisymmetric convected boundary element method formulation with uniform flow
}

\author{
BASSEM BARHOUMI ${ }^{\mathrm{a}}$ \\ University of Tunis El Manar, National Engineering School of Tunis, MAI Laboratory, BP 37, El Belvedere 1002 Tunis, Tunisia
}

Received 23 November 2015, Accepted 8 November 2016

\begin{abstract}
This paper presents an improved form of the convected Boundary Element Method (BEM) for axisymmetric problems in a subsonic uniform flow. The proposed formulation based on the axisymmetric Convected Helmholtz Equation (CHE) and its fundamental solution that describes the sound radiation from a monopole source. The variables in the new axisymmetric boundary integral formulation can be expressed explicitly in terms of the acoustic pressure and its particular normal derivative. Also, the constant coefficients are expressed only in terms of the axisymmetric convected Green's function and its convected normal derivative. The particular and convected derivatives reduce the flow effects of the normal and the flow direction derivatives incorporated in the conventional convected boundary integral formulas. The advanced form of the axisymmetric boundary integral representation with flow is a similar form of the axisymmetric boundary element method without flow. Precisely, the two new operators significantly reduce the computational burden of the classical BEM and then becomes the CPU time of BEM without flow. The formula is verified comparing to both analytical and Finite Element Methods (FEM) of an axisymmetric infinite rigid duct in a subsonic uniform flow.
\end{abstract}

Key words: Axisymmetric convected boundary element method / convected helmholtz equation / singular integrals / finite element method / axisymmetric infinite rigid duct

\section{Introduction}

The numerical studies of some acoustic problems with fluid flow require only the special methods, such as the computational techniques of the Finite Element Method (FEM) and boundary element method (BEM) for solving any axisymmetric medium in the science and engineering. The finite element method is currently the most widely used numerical method for solving the interior medium with mean flow and that the boundary element method is used for solving the exterior medium with a constant flow [1].

The finite element method has been implemented for solving the Helmholtz equation for the acoustic wave diffraction problems without flow [2]. It has been implemented with a displacement formulation for the modelling and simulation of the fluid-structure interaction problems in which the coupling conditions are formulated [3]. A numerical technique of the FEM for the modeling of the acoustic pressure inside an axisymmetric lined duct in

\footnotetext{
${ }^{a}$ Corresponding author:

bassem. barhoumi@enit.rnu.tn
}

the presence of a constant flow was developed and presented in Reference [4]. This method is based on the resolution of the convected Helmholtz equation in the modal representation which gives good results compared with the analytical ones. The FEM applied to determine the time-harmonic acoustic field in a 2D infinite waveguide with walls covered of an absorbing material, and in the presence of a mean flow assumed uniform far from the source [5]. The boundary conditions of the rigid and lined ducts are represented by transparent boundary conditions suitable for use in a finite element scheme which based on the Dirichlet-to-Neumann (DtN) boundary condition [5]. For particular acoustic domains [6], has determined the acoustic propagation in a transformed domain by the Prandtl-Glauert transformation.

It is known that the finite element method has its limitations in modeling infinite domains. The unbounded medium contains the non-reflection condition which is represented by the Sommerfeld radiation condition in a medium at rest [7] or in a subsonic uniform flow [6]. Using the boundary element method (BEM), which requires a discretization of only the generator of the acoustic domain and that this Sommerfeld condition is automatically 
fulfilled by the fundamental solution. This makes the BEM superior to the FEM in many cases, especially for the unbounded problems, such as the wave propagation in the infinite lined ducts.

The boundary element method can be formulated in the transformed acoustic medium [8] and in the original acoustic medium [9]. In order to take advantage of both the FEM and the BEM, a coupled FEM/BEM approach has been proposed [10]. A direct collocation boundary element formulation for the calculation of acoustic propagation in a subsonic uniform mean flow has been presented in Reference [11] in which the model is directed towards the calculation of the propagation inside and radiation from axisymmetric lined ducts. An advanced boundary element/fast Fourier transform (BE/FFT) methodology for solving axisymmetric acoustic wave scattering and radiation problems with non-axisymmetric boundary conditions has been developed in Reference [12].

However, the main disadvantages of using boundary integral formulations in an original acoustic medium are the same as in the non-flow case [13]. In addition, the convection effects in the presence of flow substantially increase these difficulties and makes significantly more complicated the conventional integral representations $[10,11]$. These axisymmetric formulations are derived from the three-dimensional boundary integral formulation developed in Reference [9] in which the convected terms due to the Green's function and its derivatives such as the normal derivative and the derivative in the flow direction.

Also, the authors in Reference [14] have been used a reformulation of the three-dimensional convected boundary element to solve the axisymmetric acoustic problems. The reformulation based on the non-standard normal derivative similar to the non-flow normal derivative of the Green's kernel. The non-standard derivative leads to a global form of the integral formula contains another supplementary convective terms in which make difficulty the numerical implementation of the boundary element method. The acoustic variable of the classical and reformulation axisymmetric boundary integral representations in References $[10,11,14]$ in terms of the acoustic pressure as well as its normal and tangential derivatives.

The aim of this paper is to contribute to reduce the complexity of convected boundary integral formulas for axisymmetric domains in a subsonic uniform flow. Thus, a new reformulation of the boundary element method for the axisymmetric mediums (BEMA) which based on the axisymmetric convected Helmholtz equation and its fundamental solution is presented. This new axisymmetric boundary element method requires only two operators which substantially reduce the flow effects of the normal and the flow direction derivatives of the axisymmetric convected Green's function incorporated in the classical convected boundary integral formulations. Through the use of these new convected terms, the new axisymmetric BEM formula has a similar form of the axisymmetric BEM without flow. The acoustic variables in the new axisymmetric boundary integral equation can be expressed

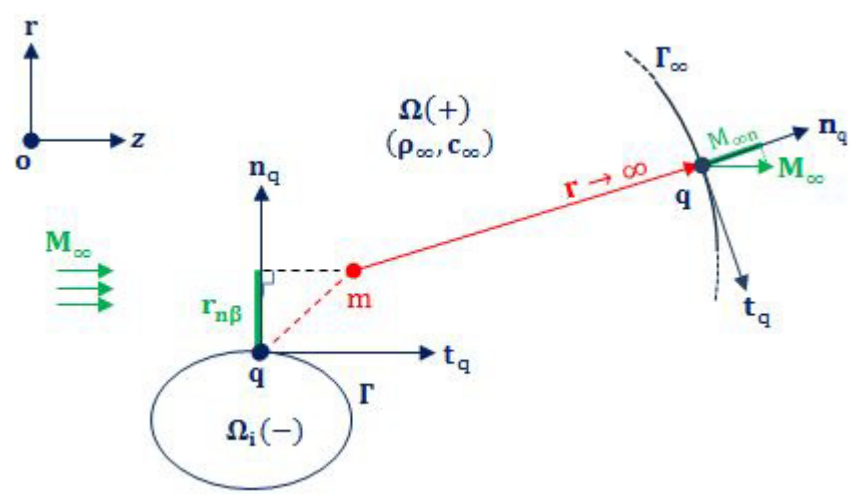

Fig. 1. Axisymmetric acoustic medium.

explicitly in terms of the acoustic pressure and its particular normal derivative.

The numerical implementation of BEMA is developed for the acoustic propagation in an axisymmetric rigid duct and validated by comparison with FEMA method and the analytical solution in which the CPU times are presented.

\section{An improved Axisymmetric BEM Formulation}

Consider an acoustic medium $\Omega$ with a compressible fluid, inviscid, isentropic, which is characterized by the Mach flow vector $\mathbf{M}_{\infty}$, of density $\rho_{\infty}$ and speed of sound $c_{\infty}$ in the axisymmetric physical space $(o, r, z)$. In Figure $1, \partial \Omega=\Gamma_{\infty} \cup \Gamma$ is the generator limiting the axisymmetric medium $\Omega$, such as $\Gamma_{\infty}$ and $\Gamma$ are the exterior and interior generators of the outgoing normal vector $\mathbf{n}_{\mathbf{q}}\left(n_{r_{q}}, n_{z_{q}}\right)$ and the tangential vector $\mathbf{t}_{\mathbf{q}}\left(t_{r_{q}}, t_{z_{q}}\right)$ at the point $q$, respectively. The generator $\partial \Omega$ not perturbed the streamlines and unchanged the direction of the flow.

\subsection{Axisymmetric convected helmholtz equation}

A combination of the Axisymmetric Linearized Euler Equations can be produced the Axisymmetric Convected Helmholtz Equation in a harmonic regime $(\exp (+i \omega t))$ in which $t$ is the time, $\omega$ is the angular frequency and $\mathrm{i}$ is the imaginary unit. The wave equation wearing on axisymmetric acoustic pressure $\mathrm{p}$ is decomposed into a non-flow part and a convection part [10]

$$
H(p)\left[\Delta+k^{2}\right](p)-\left(\mathbf{M}_{\infty} \cdot \nabla\right)\left[\left(2 i k+\left(\mathbf{M}_{\infty} \cdot \nabla\right)\right)(p)\right]=0
$$

where $\Delta$ and $\nabla$ are the axisymmetric Laplace and Gradient operators. $k=\omega / c_{\infty}$ is the original wavenumber. The fundamental solution of Equation (1) is the axisymmetric convected Green's function $G_{0}^{k}=\int_{0}^{2 \pi} G^{k} d \beta$, which is the integration of the three-dimensional convected Green function $G^{k}$ over the azimuthal angle $\beta$. The Green's kernel $G^{k}(m, q)$ is given for the points $m$ and $q$ by

$$
G^{k}(m, q)=\exp \left[-i k^{*}\left(\mathbf{m q} \cdot \mathbf{M}_{\infty}^{*}+R_{\beta}^{*}\right)\right] /\left(4 \pi \alpha R_{\beta}^{*}\right),
$$


where $m\left(r_{m}, z_{m}\right)$ and $q\left(r_{q}, z_{q}\right)$ are the axisymmetric source-observer points, $\alpha$ is the Prandtl-Glauert factor, $k^{*}=k / \alpha$ is the convected wavenumber, and that $\mathbf{M}_{\infty}^{*}=$ $\mathbf{M}_{\infty} / \alpha$ is the convected Mach vector. The distance $R_{\beta}^{*}$ is the convected radius which depends on the azimuthal order $\beta$, it's given for the points $m$ and $q$ by

$$
R_{\beta}^{*}(m, q)=\sqrt{r^{*^{2}}+4 r_{q} r_{m} \sin ^{2}(\beta / 2)},
$$

in which $r^{*}(m, q)=\sqrt{m q^{2}+\left(\mathbf{m q} \cdot \mathbf{M}_{\infty}^{*}\right)^{2}}$ is the axisymmetric convected radius and $m q$ is the physical distance between source-observer. Also, the axisymmetric convected Green's function satisfies the following inhomogeneous convected Helmholtz equation

$$
\begin{aligned}
H\left(G_{0}^{k}(m, q)\right) & =\left[\Delta_{q}+k^{2}\right] G_{0}^{k}(m, q) \\
& +\left(\mathbf{M}_{\infty} \cdot \nabla_{\mathbf{q}}\right)\left[\left(2 i k-\left(\mathbf{M}_{\infty} \cdot \nabla_{\mathbf{q}}\right)\right) G_{0}^{k}(m, q)\right] \\
& =-\frac{1}{2 \pi r_{q}} \delta(q-m),
\end{aligned}
$$

in which $(\cdot)_{q}$ is the operator at point $\mathrm{q}$ and $\delta$ is the Dirac delta function such as $\delta(q-m)=\delta\left(r_{q}-r_{m}\right) \delta\left(z_{q}-z_{m}\right)$.

\subsection{Axisymmetric convected Helmholtz integral equation}

To obtain a boundary integral equation formulation for the acoustic problem, the Helmholtz Equation (1) is multiplied by the axisymmetric convected Green's function $G_{0}^{k}(m, q)$ and its fundamental Equation (4) by $p(q)$. Subtracting these two equations and integrating over the axisymmetric exterior medium $\Omega$ yields

$$
\begin{aligned}
p(m)=\int_{\Omega}\left[G_{0}^{k}(m, q) H(p(q))\right. & \left.-p(q) H\left(G_{0}^{k}(m, q)\right)\right] \\
& \times r_{q} d \Omega_{q}, m \in \Omega \backslash \Gamma,
\end{aligned}
$$

Using the axisymmetric second Green's formula, the axisymmetric Gradient property and the axisymmetric divergence theorem (Appendix A), the axisymmetric acoustic pressure Equation (5) takes the following form

$$
\begin{aligned}
p(m) & =\int_{\partial \Omega}\left[G_{0}^{k}(m, q) \frac{\partial p}{\partial n_{q}}(q)-p(q) \frac{\partial G_{0}^{k}}{\partial n_{q}}(m, q)\right] r_{q} d \Gamma_{q} \\
& -2 i k \int_{\partial \Omega} M_{\infty n} p(q) G_{0}^{k}(m, q) r_{q} d \Gamma_{q} \\
& -\int_{\partial \Omega} M_{\infty n} G_{0}^{k}(m, q)\left(\mathbf{M}_{\infty} \cdot \nabla_{\mathbf{q}}\right) p(q) \\
& +\int_{\partial \Omega} M_{\infty n} p(q)\left(\mathbf{M}_{\infty} \cdot \nabla_{\mathbf{q}}\right) G_{0}^{k}(m, q) r_{q} d \Gamma_{q}, m \in \Omega \backslash \Gamma,
\end{aligned}
$$

where $\nabla_{\mathbf{q}}=\sigma_{n} \mathbf{n}_{\mathbf{q}}+\sigma_{t} \mathbf{t}_{\mathbf{q}}$ is the axisymmetric Gradient operator in which the couple $\left(\sigma_{n}=\partial / \partial_{n_{q}}, \sigma_{t}=\partial / \partial_{t_{q}}\right)$ designates the normal and tangential derivatives at point $q$, respectively. $M_{\infty n}=\mathbf{M}_{\infty} \cdot \mathbf{n}_{\mathbf{q}}$ is the normal Mach number.

In order to simplify the boundary integral Equation (6), the normal derivative $\partial / \partial n_{q}$ and the flow direction derivative $\left(\mathbf{M}_{\infty} \cdot \nabla_{\mathbf{q}}\right)$ can be converted into a particular normal derivative $d / d n_{q}$, which is given by the following relation

$$
\frac{d(\cdot)}{d n_{q}}=\frac{\partial(\cdot)}{\partial n_{q}}-M_{\infty n}\left(\mathbf{M}_{\infty} \cdot \nabla_{\mathbf{q}}\right)(\cdot),
$$

The particular normal derivative of the axisymmetric convected Green function $d G_{0}^{k} / d n_{q}=\int_{0}^{2 \pi} d G^{k} / d n_{q} d \beta$ is the integration of the particular normal derivative of the three-dimensional Green kernel $d G^{k} / d n_{q}$ over the azimuthal angle $\beta$. Using the normal and the flow direction derivatives of the three-dimensional Green's function in References $[10,11,14]$, one obtains the following derivative function

$$
\begin{aligned}
\frac{d G^{k}}{d n_{q}}(m, q)=-G^{k}(m, q) & {\left[\left(1+i k^{*} R_{\beta}^{*}(m, q)\right)\right.} \\
& \left.\times \frac{r_{n \beta}}{R_{\beta}^{* 2}(m, q)}+i k M_{\infty n}\right],
\end{aligned}
$$

where $r_{n \beta}=\mathbf{m q}_{\mathbf{a x i}} \cdot \mathbf{n}_{\mathbf{q}}$ is the normal distance and that the vector $\mathbf{m q a x i}$ of coordinate $\left(r_{q}-r_{m} \cos (\beta), z_{q}-z_{m}\right)$. The particular normal derivative of the convected Green's function Equation (8) is decomposed into two terms. The first term generalizes the expression of the normal derivative in a non-flow case and that the second term explains the convective influence. Substituting the particular normal derivatives Equations (7) and (8) in the integral formula Equation (6) yields

$$
\begin{aligned}
p(m)=\int_{\partial \Omega}[ & G_{0}^{k}(m, q) \frac{d p}{d n_{q}}(q) \\
& \left.\quad-p(q) \frac{d_{k} G_{0}^{k}}{d n_{q}}(m, q)\right] r_{q} d \Gamma_{q}, m \in \Omega \backslash \Gamma,
\end{aligned}
$$

The axisymmetric boundary integral formulation Equation (9) is decomposed into two integrals in which the acoustic variables are expressed only in terms of the acoustic field as well as its particular normal derivative which interpreting as a condition applied to the interior generator $\Gamma$. Thus, the axisymmetric BEM Equation (9) is similar to the boundary integral equation solution for solving the axisymmetric potential flows [15] and the nonflow case (the right first term in the Equation (6)) [16]. From the particular normal derivative operator Equation (7), we obtain the convected normal derivative operator

$$
\frac{d_{k}(\cdot)}{d n_{q}}=\frac{d(\cdot)}{d n_{q}}+2 i k M_{\infty n}(\cdot),
$$


Substituting the particular normal derivative of the threedimensional Green's function Equation (8) into the convected normal derivative operator Equation (10), one obtains

$$
\begin{aligned}
\frac{d_{k} G^{k}}{d n_{q}}(m, q)=-G^{k}(m, q) & {\left[\left(1+i k^{*} R_{\beta}^{*}(m, q)\right)\right.} \\
\times & \left.\frac{r_{n \beta}}{R_{\beta}^{* 2}(m, q)}-i k M_{\infty n}\right],
\end{aligned}
$$

The integration over the circular generator $\Gamma_{\infty}$ given by Equation (9), represents contributions waves which come in the interior region due to the source is very far from observer point; $q \rightarrow \infty$. Thus, this integral is null. According to the three-dimensional Green's function at infinity [17], the axisymmetric Green's kernel $G_{0}^{k}$ is proportional to $1 / \sqrt{R_{\beta}^{*}}$. Also, taking into account the term $r_{n \beta} / R_{\beta}^{*}$ is equivalent to the tangential PrandtlGlauert factor $\beta_{t}^{*}=\beta / \sqrt{1-M_{\infty t}^{2}}$, the convected normal derivative $d G_{0}^{k} / d n_{q}$ of Equation (11) is proportional to $i k\left(1 / \sqrt{1-M_{\infty t}^{2}}-M_{\infty n}\right) / \sqrt{R_{\beta}^{*}}$. This leads to two convected radiation conditions which verified by the axisymmetric acoustic pressure and its Green's kernel

$$
\begin{gathered}
\lim _{r \rightarrow \infty} p(r)=0, \\
\lim _{r \rightarrow \infty}\left\{\sqrt{r}\left[\frac{d p}{d r}(r)+i\left(\frac{k}{\sqrt{1-M_{\infty t}^{2}}}-k M_{\infty n}\right) p(r)\right]\right\}=0,
\end{gathered}
$$

where $M_{\infty t}=\mathbf{M}_{\infty} \cdot \mathbf{t}_{\mathbf{q}}$ is the tangential Mach number. The first condition Equation (12) indicates the non-reflection of waves, and the second condition Equation (13) generalizes the classical Sommerfeld radiation condition in a uniform flow subsonic $[7,8]$. Substituting the non-reflection conditions into the boundary Equation (9), we obtain

$$
\begin{array}{r}
p(m)= \pm \int_{\Gamma}\left[G_{0}^{k}(m, q) \frac{d p}{d n_{q}}(q)-p(q) \frac{d_{k} G_{0}^{k}}{d n_{q}}(m, q)\right] \\
\times r_{q} d \Gamma_{q}, m \in \Omega \backslash \Gamma, \quad
\end{array}
$$

in which the signs $(+,-)$ designates the exterior and interior acoustic domains. When the point $\mathrm{m}$ is taken on the generator at point $q$, the Green's kernel and its convected normal derivative Equations (2) and (11) contain singular and regular parts. Thus, Equation (14) requires to study of behavior of the kernels near the singularity.

\subsection{Singular integrals}

A classical procedure can be used to isolate the singularity problems of the axisymmetric Green's function and its convected derivative. In fact, the singularities can be isolated by decomposing the axisymmetric convected Green's function $G_{0}^{k}$ into a singular static part $G_{0}^{0}(k=0)$ and regular part $g_{0}^{k}$ depending on the wavenumber. Then, the axisymmetric Green's function takes the following form

$$
G_{0}^{k}(m, q)=G_{0}^{0}(m, q)+g_{0}^{k}(m, q)
$$

Thus, the convected normal derivative is given by

$$
\frac{d_{k} G_{0}^{k}}{d n_{q}}(m, q)=\frac{d_{k} G_{0}^{0}}{d n_{q}}(m, q)+\frac{d_{k} g_{0}^{k}}{d n_{q}}(m, q),
$$

Because the difference between the singular and regular behavior, the singular functions in Equations (15) and (16) are evaluated analytically and that the regular parts are evaluated numerically with standard GaussLegendre quadrature of high order. In fact, the static axisymmetric Green's function is given by $[10,11]$

$$
G_{0}^{0}(m, q)=\frac{K(\tau)}{\pi \alpha \tau^{*}}
$$

Using the normal and the flow direction derivatives of the static Green's kernel in Reference [10], one obtains the following static convected derivative

$$
\begin{aligned}
\frac{d_{k} G_{0}^{0}}{d n_{q}}(m, q)=-\frac{1}{\pi \alpha \tau^{*}} & {\left[\left(\frac{r_{n \pi / 2}}{r^{* 2}}-\frac{n_{r_{q}}}{2 r_{q}}\right) E(\tau)\right.} \\
+ & \left.\left(\frac{n_{r_{q}}}{2 r_{q}}-i k M_{\infty n}\right) K(\tau)\right]
\end{aligned}
$$

where $K(\tau)$ and $E(\tau)$ are the complete elliptic integrals of the first and second kinds, the parameters $\tau$ and $\tau^{*}$ are given by

$$
\tau^{2} \tau^{* 2}=4 r_{q} r_{m}, \tau^{*}=\sqrt{r^{* 2}+4 r_{q} r_{m}}
$$

According to the complete elliptic integrals at $r \rightarrow 0$ [17] , the static Green's function exhibits a logarithmic behavior near the singularity, as follows

$$
G_{0}^{0}(m, q) \sim-\frac{1}{2 \pi \alpha} \frac{\log \left(r^{*}\right)}{2 \pi r_{q}}, r \rightarrow 0,
$$

Thus, the static convected normal derivative Equation (18) near the singularity is characterized by

$$
\frac{d_{k} G_{0}^{0}}{d n_{q}}(m, q) \sim-\frac{1}{2 \pi \alpha} \frac{1}{2 \pi r_{q}} \frac{r_{n 0}}{r^{*^{2}}}, r \rightarrow 0,
$$

Since $r_{n 0} / r^{*}=O\left(r^{*}\right)$, then the singularity of the static convected function $d_{k} G_{0}^{k} / d n_{q}$ is thus only apparent. Following the exclusion procedure $[18,19]$ to isolate the singularity problems in the boundary formula Equation (14), 


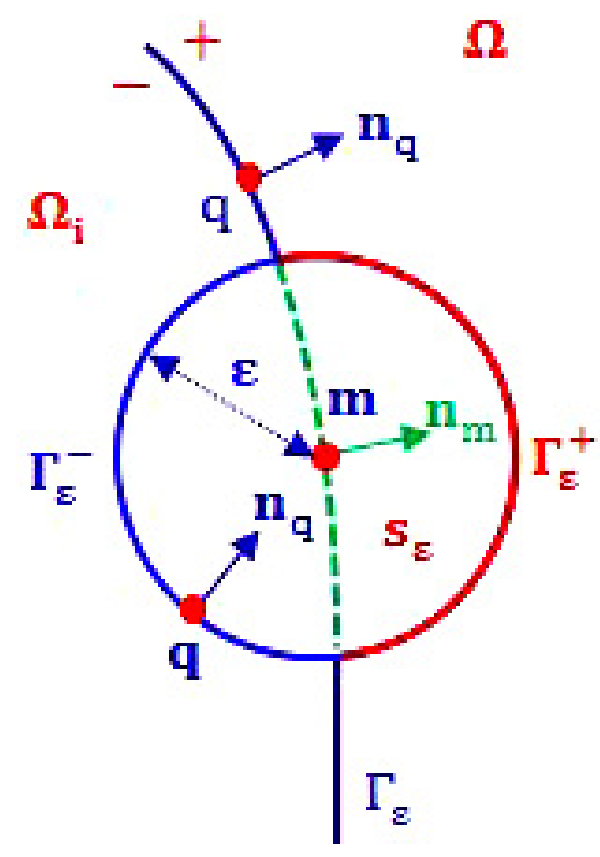

Fig. 2. Analytical regular method.

which can be rewritten as

$$
\begin{aligned}
p(m) & = \pm \lim _{\varepsilon \rightarrow 0} \int_{\Gamma_{\varepsilon}}\left[G_{0}^{k}(m, q) \frac{d p}{d n_{q}}(q)\right. \\
& \left.-p(q) \frac{d_{k} G_{0}^{k}}{d n_{q}}(m, q)\right] r_{q} d \Gamma_{q} \\
& \pm \lim _{\varepsilon \rightarrow 0} \int\left[G_{\Gamma_{\varepsilon}^{\mp}}^{k}(m, q) \frac{d p}{d n_{q}}(q)-p(q) \frac{d_{k} G_{0}^{k}}{d n_{q}}(m, q)\right] r_{q} d \Gamma_{q},
\end{aligned}
$$

where $s_{\varepsilon}$ is the disk of center $\mathrm{m}$ and of radius $\varepsilon=r$ bounded by the circle $\Gamma_{\varepsilon}^{+} \cup \Gamma_{\varepsilon}^{-}$and $\Gamma=\lim _{\varepsilon \rightarrow 0}\left(\Gamma_{\varepsilon} \cup \Gamma_{\varepsilon}^{-}\right)$ for the exterior domain and that $\Gamma=\lim _{\varepsilon \rightarrow 0}\left(\Gamma_{\varepsilon} \cup \Gamma_{\varepsilon}^{+}\right)$for the interior domain (see Fig. 2).

Substituting Equations (20) and (21) in Equation (22), we get a new original form of the integral boundary representation with uniform flow wearing on the axisymmetric acoustic pressure at any point $m$

$$
\begin{aligned}
& c^{ \pm}(m) p(m)= \pm \int_{\Gamma} G_{0}^{k}(m, q) \frac{d p}{d n_{q}}(q) r_{q} d \Gamma_{q} \\
& \mp \int_{\Gamma} p(q) \frac{d_{k} G_{0}^{k}}{d n_{q}}(m, q) r_{q} d \Gamma_{q}, m \in \Omega,
\end{aligned}
$$

where $c^{ \pm}(m)$ is the free term at any point $m$, which is given by

$$
c^{ \pm}(m)=1-\frac{\theta^{\mp}(m)}{2 \pi}=1 \pm \int_{\Gamma}-\frac{1}{2 \pi \beta} \frac{\mathbf{m q} \cdot \mathbf{n}_{\mathbf{q}}}{r^{*^{2}}(m, q)} d \Gamma_{q},
$$

in which $\theta^{ \pm}(m)$ is the convected angle. When the point $\mathrm{m}$ in the exterior and interior domains, the convected angle $\theta^{ \pm}(m)=0$ and that the coefficient $c^{ \pm}(m)=1$. If $\mathrm{m}$ is a regular point of the generator, $\theta^{ \pm}(m)=\pi$ and that $c^{ \pm}(m)=0.5$.

\subsection{Numerical implementation}

The numerical implementation of the axisymmetric boundary integral Equation (23) for a body of arbitrary shape is obtained by discretizing the generator $\Gamma$ of the body with $N$ one-dimensional quadratic isoparametric elements, according to the following convergence criterion

$$
n_{e} h_{e} \leq \lambda \alpha^{2}
$$

where $n_{e}$ is the number of finite elements for a wavelength $\lambda$ and $h_{e}$ is the diameter of the finite element. Then, by using the quadratic shape functions, the acoustic pressure $p$ and its normal derivative $\sigma_{n}$ of any point $q$ on the generator $\Gamma$ are assumed to be given in terms of the nodal coordinates. Thus, the tangential derivative in the local coordinates system along the generator $\Gamma$ at point $q$ is given by $[11,20]$

$$
\sigma_{t}(q)=\sigma_{\xi}(q) / J^{i}[\xi(q)]
$$

with $-1 \leq \xi \leq 1$ is the local coordinate and $J^{i}$ is the Jacobien of reference-local transformation. Using the collocation method so that the point $m$ coincides with the nodes $q_{i}$ of the discretized generator $\Gamma$, Equation (14) can be written in complex matrix form with Robin condition

$$
[\mathbf{A}] \mathbf{p}=\left[\mathbf{B}_{\mathbf{n}}\right] \sigma_{\mathbf{n}}+\left[\mathbf{B}_{\mathbf{c}}\right] \mathbf{p}_{\mathbf{c}}
$$

where $\mathbf{p}, \mathbf{p}_{\mathbf{c}}$ and $\sigma_{\mathbf{n}}$ are the nodal vectors containing the values of the axisymmetric acoustic pressure $p$, its Dirichlet acoustic pressure $p_{c}$ and its Neumann normal derivative $\sigma_{n}$ at each node, respectively, whereas $[\mathbf{A}]$ and $\left[\mathbf{B}_{\mathbf{n}}\right]$ are the axisymmetric acoustic matrices with the elements of matrix $[\mathbf{A}]$ contain the convected angles and elementary integrals related to the axisymmetric convected Green's function $G_{0}^{k}$ and its convected normal derivative $d_{k} G_{0}^{k} / d n_{q}$, while the elements of matrix $\left[\mathbf{B}_{\mathbf{n}}\right]$ is composed only by the elementary integrals containing the integrands of axisymmetric Green's function and its convected derivative. $\left[\mathbf{B}_{\mathbf{c}}\right]$ is the acoustic matrix containing the integrands $G_{0}^{k}$ and $d_{k} G_{0}^{k} / d n_{q}$.

\section{Axisymmetric finite element method}

The axisymmetric Finite Element Method is based on the weak variational formulation, which can be obtained by multiplying the axisymmetric wave Equation (1) by a test function $p^{*}$ (the axisymmetric acoustic pressure $p^{*}$ satisfies the axisymmetric convected Helmholtz Eq. (1)). 
Then, integrating the result over the axisymmetric interior medium $\Omega_{i}$, one obtains

$$
\begin{aligned}
\int_{\Omega_{i}} p^{*} \Delta p d \Omega_{i}+k^{2} \int_{\Omega_{i}} p^{*} p d \Omega_{i} & -2 i k M_{\infty} \int_{\Omega_{i}} p^{*} \frac{\partial p}{\partial z} d \Omega_{i} \\
& -M_{\infty}^{2} \int_{\Omega_{i}} p^{*} \frac{\partial^{2} p}{\partial z^{2}} d \Omega_{i}
\end{aligned}
$$

Using the axisymmetric second Green's formula, one obtains

$$
\begin{gathered}
\int_{\Omega_{i}}\left[p^{*} \Delta p\right] r d \Omega_{i}=-\int_{\Omega_{i}} \nabla p^{*} \nabla p r d \Omega_{i}+\int_{\Gamma_{i}} p^{*} \frac{\partial p}{\partial n_{\Gamma_{i}}} r d \Gamma_{i} \\
\int_{\Omega_{i}}\left[p^{*} \frac{\partial^{2} p}{\partial z^{2}}\right] r d \Omega_{i}=-\int_{\Omega_{i}} \frac{\partial p^{*}}{\partial z} \frac{\partial p}{\partial z} r d \Omega_{i}+\int_{\Gamma_{i}} p^{*} \frac{\partial p}{\partial n_{z}} r d \Gamma_{i}
\end{gathered}
$$

$$
\begin{aligned}
2 \int_{\Omega_{i}} p^{*} \frac{\partial p}{\partial z} r d \Omega_{i}=\int_{\Omega_{i}} p^{*} \frac{\partial p}{\partial z} r d \Omega_{i} & -\int_{\Omega_{i}} \frac{\partial p^{*}}{\partial z} p r d \Omega_{i} \\
& +\int_{\Gamma_{i}} p^{*} p n_{z} r d \Gamma_{i}
\end{aligned}
$$

Substituting Equations (29)-(31) into Equation (28), we obtain a developed form of the weak variational formulation in References $[4,21]$

$$
\begin{aligned}
& -\int_{\Omega_{i}} \nabla p^{*} \nabla p r d \Omega_{i}+k^{2} \int_{\Omega_{i}} p^{*} p r d \Omega_{i}+i k M_{\infty} \int_{\Omega_{i}} p^{*} \frac{\partial p}{\partial z} r d \Omega_{i} \\
& -i k M_{\infty} \int_{\Omega_{i}} \frac{\partial p^{*}}{\partial z} p r d \Omega_{i}+M_{\infty}^{2} \int_{\Omega_{i}} \frac{\partial p^{*}}{\partial z} \frac{\partial p}{\partial z} r d \Omega_{i} \\
& +\int_{\Gamma_{i}} p^{*} \frac{\partial p}{\partial n_{\Gamma_{i}}} r d \Gamma_{i}+i k M_{\infty} \int_{\Gamma_{i}} p^{*} p n_{z} r d \Gamma_{i} \\
& -M_{\infty}^{2} \int_{\Gamma_{i}} p^{*} \frac{\partial p}{\partial n_{z}} r d \Gamma_{i}=0,
\end{aligned}
$$

The same classical numerical discretization in Reference [4], the elementary integrals of the numerical variational formulation Equation (32) over the reference triangular or interval elements $\hat{T}$ take the following form

$$
\int_{\hat{T}} f * P d \hat{T},
$$

in which $f$ and $P$ are the regular polynomials of order $n(n<3)$ containing the shape functions. Then, these integrals are evaluated analytically [22]. Thus, the solve equation is a similar form of the complex matrix form Equation (27) in which $[\mathbf{A}],\left[\mathbf{B}_{\mathbf{n}}\right]$ and $\left[\mathbf{B}_{\mathbf{c}}\right]$ are the axisymmetric acoustic matrices with the various integrals in Equation (32).

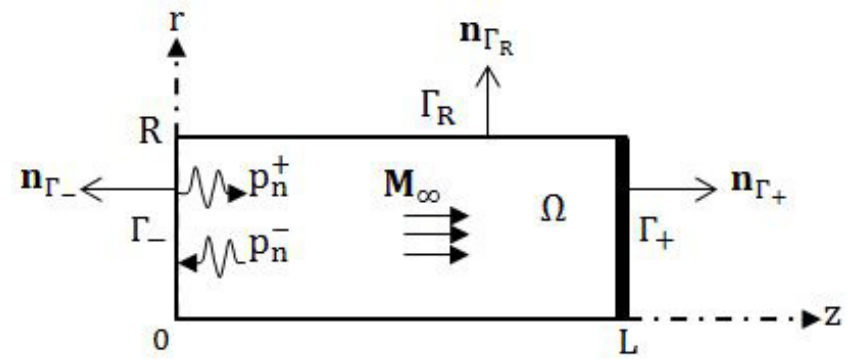

Fig. 3. Axisymmetric rigid duct.

\section{Application: Axisymmetric rigid duct}

We consider an infinite axisymmetric rigid duct of truncated domain $\Omega$ of radius $R=0.1 \mathrm{~m}$, of length $L=0.5 \mathrm{~m}$, of symmetry axis $(o z)$, in a subsonic uniform flow of the Mach vector $\mathbf{M}_{\infty}=M_{\infty} \mathbf{e}_{\mathbf{z}}$ in the $(r-z)$ meridian plane. The truncated medium is limited by the rigid wall $\Gamma_{R}$ of the normal vector $\mathbf{n}_{\Gamma_{\mathbf{R}}}$ and by two generators, the left boundary $\Gamma_{-}$at $z=0$ and the right boundary $\Gamma_{+}$at $z=L$ of normal vectors $\mathbf{n}_{\boldsymbol{\Gamma}_{-}}$and $\mathbf{n}_{\boldsymbol{\Gamma}_{+}}$, respectively (see Fig. 3).

The acoustic pressure in an axisymmetric rigid duct is $[3]$

$$
p_{n}^{ \pm}=A_{n} J_{0}\left(k_{r, n} r\right) e^{i k_{z, n}^{ \pm} z},
$$

in which $(0)$ is the azimuthal order and $(n)$ is the radial order, $A_{n}$ is the axisymmetric amplitude of order $n$ equal to $\frac{\sqrt{2}}{R} \frac{1}{\sqrt{J_{0}^{2}\left(k_{r, n} R\right)-J_{1}^{2}\left(k_{r, n} R\right)}}, J_{0}$ is the Bessel function of the first kind and that $k_{z, n}^{ \pm}$and $k_{r, n}$ are the axial and radial wavenumbers ( \pm indicates the propagation mode in the increased $(+)$ and decreased (-) z-direction) which satisfies the following relations

$$
\begin{aligned}
\left.\frac{\partial J_{0}\left(k_{r, n} r\right)}{\partial r}\right|_{r=R} & =0, \\
k_{z, n}^{ \pm} & =\frac{-k M_{\infty} \pm \sqrt{k^{2}-\alpha^{2} k_{r, n}^{2}}}{\alpha^{2}},
\end{aligned}
$$

Since the term as the function of tangential derivative $\sigma_{t}$ over the axisymmetric generator duct is zero, then the new radiation condition is given by the following particular derivative $\sigma$ at right and left generators $\Gamma_{ \pm}$

$$
\sigma_{ \pm}= \pm i \alpha^{2} k_{z, n}^{ \pm} p
$$

We note $k_{n}^{ \pm}=\alpha^{2} k_{z, n}^{ \pm}$is the new wavenumber in which its module reduces the module of the classical wavenumber $k_{z, n}^{ \pm}$for the axisymmetric excitations.

\subsection{Numerical methodologies}

The two-dimensional rigid duct is discretized by triangular elements structured and regular with three nodes isoparametric of two straight perpendiculars. 
Table 1. The CPU times of BEM, Classical BEM and FEM.

\begin{tabular}{cccc}
\hline Mach & Presented & Classical & Presented \\
vector & BEM & BEM & FEM \\
\hline $\mathbf{M}_{\infty}=\mathbf{0}$ & $2.71 \mathrm{~min}$ & $3.08 \mathrm{~min}$ & $8.45 \mathrm{~min}$ \\
$\mathbf{M}_{\infty}=0.4 \mathbf{e}_{\mathbf{z}}$ & $2.73 \mathrm{~min}$ & $6.92 \mathrm{~min}$ & $13.86 \mathrm{~min}$ \\
\hline
\end{tabular}

The axial and radial wavenumbers satisfies the convergence criterion of the discretization Equation (25) in which the number of triangles is 1652 elements (see Fig. 4).

The evaluation of the acoustic pressure at point $\mathrm{m}$ in an interior axisymmetric rigid duct $\Omega$ given by Equation (23) is numerically where $\Gamma=\Gamma_{-} \cup \Gamma_{R} \cup \Gamma_{+}$is the generator of this duct, and taking into account that the free term at the corner points $m=\Gamma_{-} \cap \Gamma_{R}$ and $m=\Gamma_{R} \cap \Gamma_{+}$ is $c^{-}(m)=3 / 4$.

\subsection{Numerical results}

We apply BEMA, FEMA techniques and the analytical solution (S.Anal) associated to the axisymmetric acoustic pressure which propagated in the increased $z$ direction of axisymmetric excitation $\left(p_{n}^{+} / A_{n}\right)$. Figures 5 and 6 give the cartographies of the real parts $\operatorname{Re}\left(P_{n}^{+} / A_{n}\right)$ for different values of the Mach vector by S.BEMA, S.FEMA and S.Anal methods.

Figures 5 and 6 are presented the real parts of the axisymmetric acoustic pressure $\operatorname{Re}\left(P_{n}^{+} / A_{n}\right)$ by FEMA and BEMA which are in very good concord with analytical solution of relative error given by the $L_{2}$ norm. For FEMA, the relative error is less than $0.5 \%$ and for the axisymmetric boundary integral method, the error is less than $0.8 \%$.

We observe that for the initial mode (0), the axisymmetric acoustic pressure is uniform along the radial and axial waves. Also, when the subsonic uniform flow is increased, the wavelength increases, which is explained by the offset of the wave in the positive $z$-direction (see Fig. 5).

In the other mode, the axisymmetric acoustic pressure along the radial and axial axis is non-uniform in the presence and absence of the flow. Indeed, in the upstream region, the acoustic field is radially amplified to be greater than that obtained in the downstream region in which we observe the Sommerfeld radiation condition Equation (12), and we notice also that the uniform flow is proportional to the wavelength (see Fig. 6).

In addition, the CPU times of the Numerical boundary element method, the finite element method and that the classical boundary element method in Reference [14] with mean flow are obtained by a machine $2.93 \mathrm{GHz}$ using MATLAB-ACOUSTIC codes, which are given by the following Table 1.

A comparison between the CPU times of FE and BE methods in Table 1, show that the axisymmetric Convected boundary element formulation Equation (23) reduces $80 \%$ of the computation time of Finite element method Equation (32).
Compared to the classical axisymmetric boundary integral formulae, the proposed method reduces a CPU time equal to $60 \%$ of the classical axisymmetric BEM. Also, the relative error for the classical axisymmetric boundary integral equation is less than $2 \%$.

We observe that when the number of the discretization increase in term of the numerical implementation of the axisymmetric boundary element method with mean flow, the gain time between BEM-FEM and BEM-Classical BEM increase.

For the BEM case, we observe that the numerical execution time for the non-flow case is the numerical execution time for the flow case. This is because the improved form of the axisymmetric boundary integral representation Equation (23) with the mean flow is similar to the boundary element method without flow.

\section{Conclusion and prospects}

In this work, an improved form of the axisymmetric Helmholtz integral equation and conventional finite element method for an axisymmetric medium in a subsonic uniform flow have been developed and presented. The axisymmetric convected boundary element method is derived from two new operators such as the convected and particular derivatives, which substantially reduces the complexity due to the presence of flow in the conventional formulations. The formulation is derived to be easy to implement as a numerical tool for computational codes of the axisymmetric acoustic medium in a mean flow.

Also, the improved boundary integral representation significantly reduces the computational burden of the convenctional axisymmetric finite element method. Precisely, for the rigid duct case, the axisymmetric BEM require three Matrices in terms of integrals over the generator and that axisymmetric FEM require six assembly matrices in terms of integrals over the medium and its generator. The proposed methodologies can be applied to simplified the modal boundary element method and its derivatives $[11,23]$.

Finally, we think that the method could be extended for the analysis of physical phenomena in other noise pollution systems such as compressors, aircraft engines, and ventilation systems that contain different materials. It is hoped that this could have a significant impact in design optimization of flow duct systems in the industry sector.

\section{Appendix A. Axisymmetric acoustic properties}

The axisymmetric Green's identity formula as a function of the test function $p^{*}$ is given by

$$
\begin{aligned}
& \int_{\Omega}\left(\Delta_{q} p^{*}(q) p(q)-\Delta_{q} p(q) p^{*}(q)\right) d \Omega_{q} \\
& =\int_{\partial \Omega}\left[p^{*}(q) \frac{\partial p}{\partial n_{q}}(q)-p(q) \frac{\partial p^{*}}{\partial n_{q}}(q)\right] r_{q} d \Gamma_{q},
\end{aligned}
$$


B. Barhoumi: Mechanics \& Industry 18, 313 (2017)

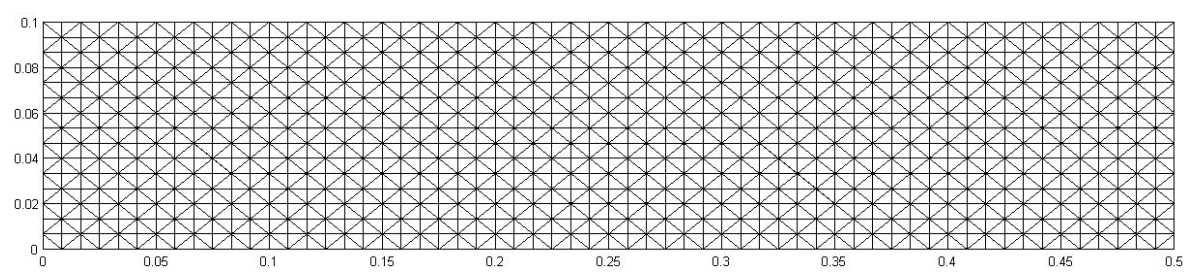

Fig. 4. Axisymmetric mesh.
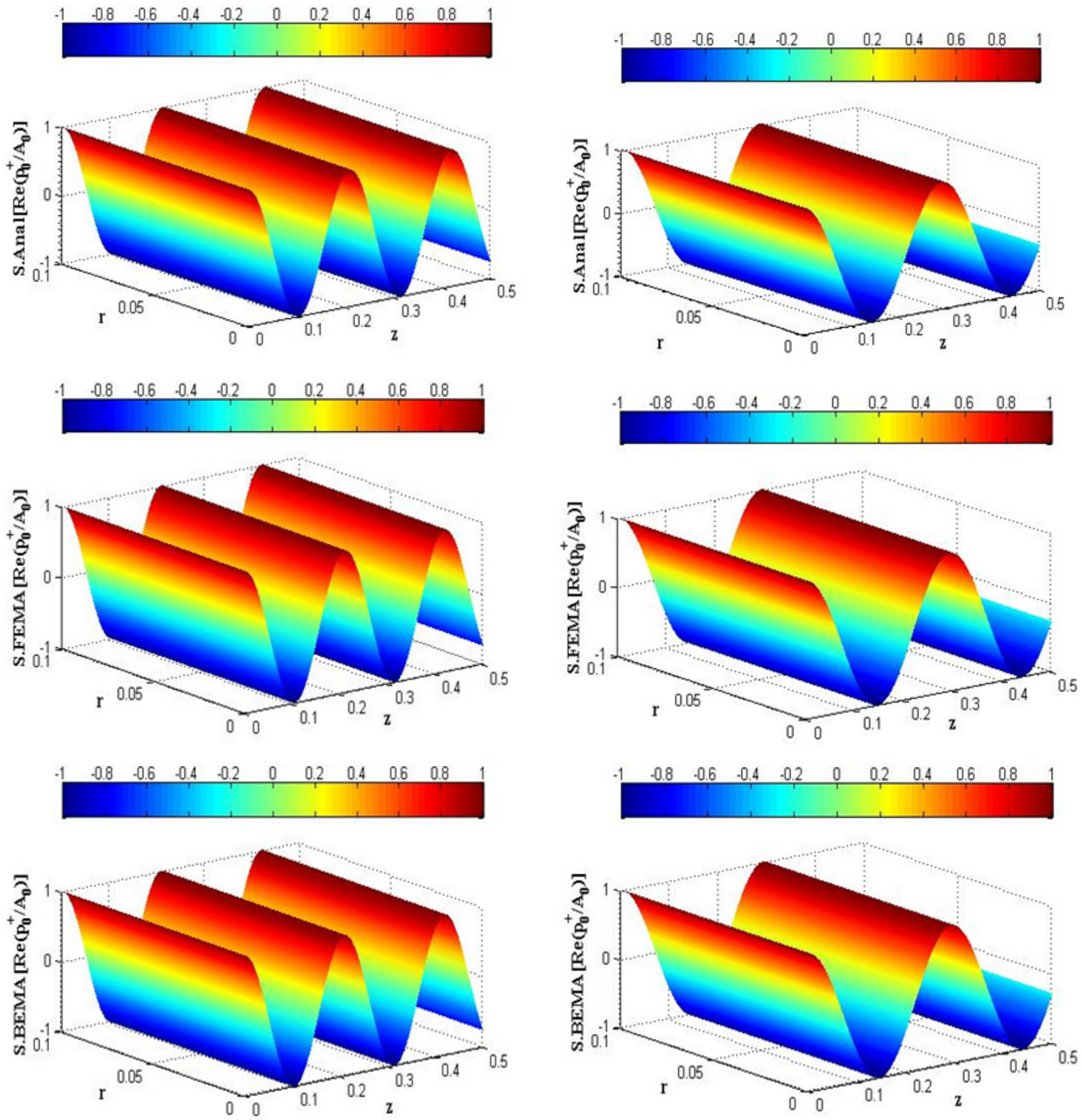

Fig. 5. Real part of the axisymmetric acoustic pressure $R e\left(P_{0}^{+} / A_{0}\right)$ by S.Anal (top), FEMA (middle) and BEMA (bottom) for $\mathbf{M}_{\infty}=\mathbf{0}$ (left) and $\mathbf{M}_{\infty}=0.4 \mathbf{e}_{\mathbf{z}}$ (right) with $k=30$. 

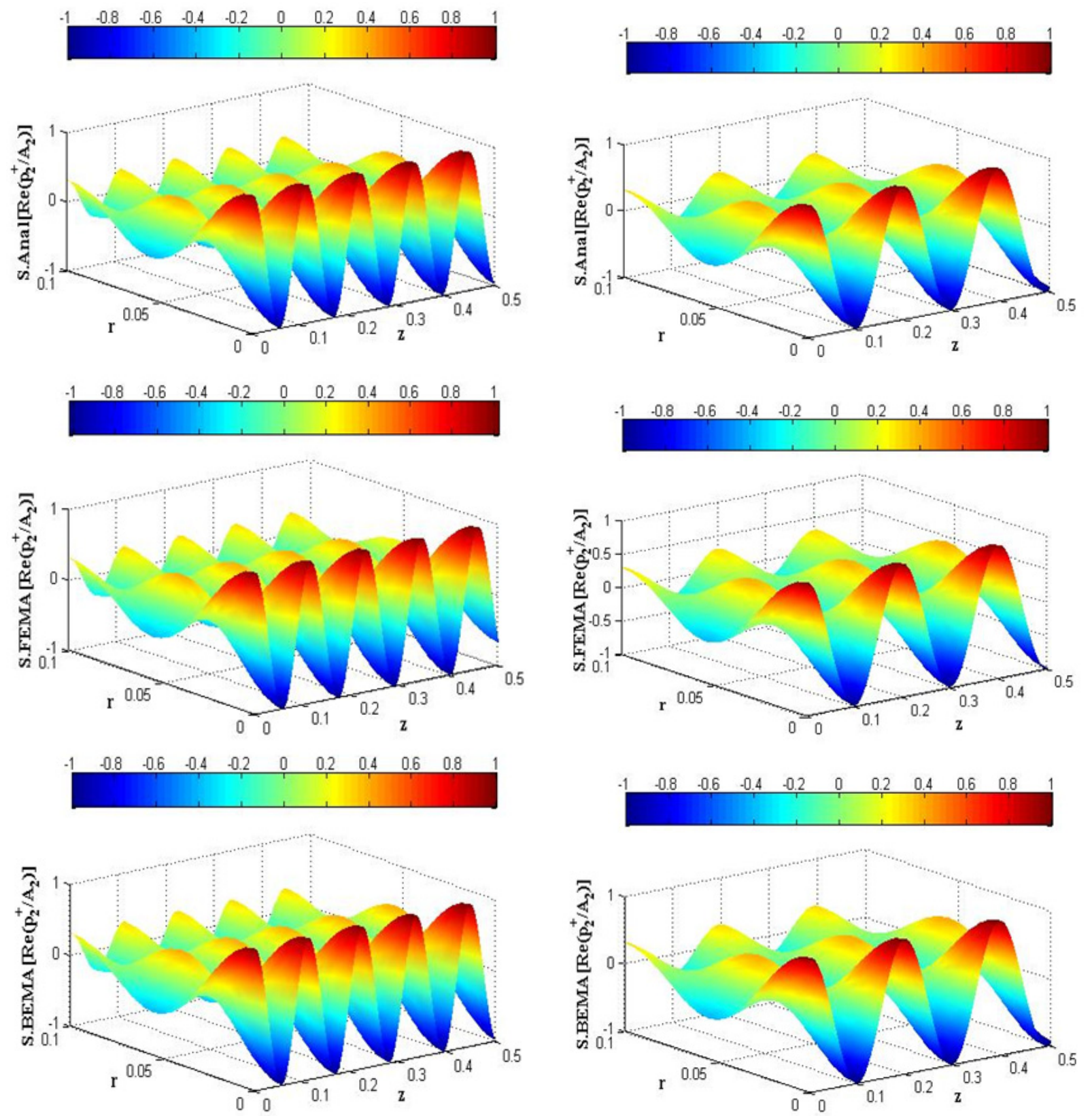

Fig. 6. Real part of the axisymmetric acoustic pressure $R e\left(P_{2}^{+} / A_{2}\right)$ by S.Anal (top), FEMA (middle) and BEMA (bottom) for $\mathbf{M}_{\infty}=\mathbf{0}$ (left) and $\mathbf{M}_{\infty}=0.4 \mathbf{e}_{\mathbf{z}}$ (right) with $k=90$.

where $\partial / \partial_{n_{q}}$ is the normal derivative and that $\Delta_{q}$ is the axisymmetric Laplace operator in an acoustics problem $r-z$

$$
\Delta_{q}(\cdot)=\frac{\partial^{2}}{\partial r_{q}^{2}}(\cdot)+\frac{1}{r_{q}} \frac{\partial}{\partial r_{q}}(\cdot)+\frac{\partial^{2}}{\partial z_{q}^{2}}(\cdot),
$$

Using the axisymmetric Green's identity formula Equation (A.1) for the axisymmetric Green's function
$G_{0}^{k}(m, q)$, yields

$$
\begin{aligned}
& \int_{\Omega}\left(\Delta_{q} G_{0}^{k}(m, q) p(q)-\Delta_{q} p(q) G_{0}^{k}(m, q)\right) d \Omega_{q} \\
= & \int_{\partial \Omega}\left[G_{0}^{k}(m, q) \frac{\partial p}{\partial n_{q}}(q)-p(q) \frac{\partial G_{0}^{k}}{\partial n_{q}}(m, q)\right] r_{q} d \Gamma_{q},
\end{aligned}
$$

The axisymmetric Gradient property is given by

$$
\begin{aligned}
& G_{0}^{k}(m, q)\left(\mathbf{M}_{\infty} \cdot \nabla_{\mathbf{q}}\right) p(q)+p(q)\left(\mathbf{M}_{\infty} \cdot \nabla_{\mathbf{q}}\right) G_{0}^{k}(m, q) \\
& =\left(\mathbf{M}_{\infty} \cdot \nabla_{\mathbf{q}}\right)\left(p(q) G_{0}^{k}(m, q)\right),
\end{aligned}
$$


And that the axisymmetric Divergence theorem is given by

$$
\begin{aligned}
& \left(p(q) \mathbf{M}_{\infty}\right) \cdot \nabla_{q}\left(\left(\mathbf{M}_{\infty} \cdot \nabla_{\mathbf{q}}\right) G_{0}^{k}(m, q)\right) \\
& =\nabla_{q} \cdot\left[\left(\left(\mathbf{M}_{\infty} \cdot \nabla_{\mathbf{q}}\right) G_{0}^{k}(m, q)\right) p(q) \mathbf{M}_{\infty}\right] \\
& -\left(\left(\mathbf{M}_{\infty} \cdot \nabla_{\mathbf{q}}\right) G_{0}^{k}(m, q)\right)\left(\left(\mathbf{M}_{\infty} \cdot \nabla_{\mathbf{q}}\right) p(q)\right),
\end{aligned}
$$

\section{References}

[1] R.J. Astley, Numerical methods for noise propagation in moving flows, with application to turbofan engines, Acoust. Sci. Tech. 30 (2009) 227-239

[2] R. Kechroud, Preconditioning techniques for the solution of the Helmholtz equation by the finite element method, Math. Comput. Simulation 65 (2004) 303-321

[3] G. Gabard, F. Treyssede, M.B. Tahar, A numerical method for vibro-acoustic problems with sheared mean flows, J. Sound Vib. 272 (2004) 991-1011

[4] M. Taktak, Numerical modelling of the acoustic pressure inside an axisymmetric lined Flow Duct, JSV 37 (2012) $151-160$

[5] E. Redon, A.-S. Bonnet-Ben Dhia, J.F. Mercier, S.P. Sari, Non-reecting boundary conditions for acoustic propagation in ducts with acoustic treatment and mean flow, Int. J. Numer. Meth. Eng. 86 (2011) 1360-1378

[6] N. Balin, F. Casenave, F. Dubois, Boundary Element and Finite Element Coupling for Aeroacoustics Simulations, JCP 294 (2015) 274-296

[7] A. Sommerfeld, Partial differential equations in physics, Publishers New York, 1949

[8] F. Casenave, A. Ern, G. Sylvand, Coupled BEM-FEM for the convected Helmholtz equation with non-uniform flow in a bounded domain, JCP 257 (2014) 627-644

[9] T.-W. Wu, L. Lee, A direct boundary integral formulation for acoustic radiation in a subsonic uniform flow, JSV 175 (1994) 51-63

[10] P. Zhang, T.-W. Wu, L. Lee, A coupled FEM/BEM formulation for acoustic radiation in a subsonic non-uniform flow, JSV 206 (1997) 309-326
[11] P. Juhl, Non-axisymmetric acoustics propagation in and radiation from lined ducts in a subsonic uniform mean flow, Acustica-acta acustica 86 (2000) 860-869

[12] V.-T. Stephanos et al., An advanced boundary element/fast Fourier transform axisymmetric formulation for acoustic radiation and wave scattering problems, J. Acoust. Soc. Am. 105 (1999) 1517-1526

[13] P.M. Juhl, An axisymmetric boundary element formulation of sound wave propagation in fluids including viscous and thermal losses, J. Acoust. Soc. Am. 135 (2013) 34093418

[14] M. Beldi, B. Barhoumi, A simple and effective axisymmetric convected Helmholtz integral equation, C.R. Mcanique 343 (2015) 457-470

[15] G.-P. Lennon, Boundary Integral Equation solution to axisymmetric potential flows, Water Resources Res. 15 (1979) 1102-1106

[16] S. Kirkup, The Boundary Element Method in Acoustics, Journal of Computational Acoustics, 2007

[17] M. Abramowitz, I.-A. Stegun, Handbook of Mathematical Functions, Dover, New York, 1972

[18] M. Bonnet, Boundary Integral Equation Methods for Solids and Fluids. John Wiley and Sons, 1999

[19] M. Guiggiani, A. Gigante, A General Algorithm for Multidimensional Cauchy Principal Value Integrals in the boundary Element Method, Appl. Mech. 57 (1990) 906915

[20] S. Mancini, R.J. Astley, S. Sinayoko, G. Gabard, An integral formulation for wave propagation on weakly nonuniform potential flows, J. Sound Vib. 385 (2016) 184-201

[21] J.N. Reddy, An Introduction to Finite Element Methods, Second Edition, McGraw Hill Inc. New York, 1993

[22] G. Dhatt, G. Touzot, Presentation of the finite elements method, Maloine S.A. Editeur, Paris, 1989

[23] P. Zhang, T.W. Wu, A hypersingular integral formulation for acoustic radiation in moving flows, J. Sound Vib. 206 (1997) 309-326 